\title{
Horse-related spine and spinal cord injuries
}

\author{
Antonios G Angoules ${ }^{1}$, Anna Christakou ${ }^{2}$, Haridimos Tsibidakis ${ }^{3}$, Georgios A Angoules ${ }^{4}$ and Stylianos Kapetanakis ${ }^{5}$ \\ ${ }^{1}$ Orthopaedic Department, Athens Medical Center, Athens, Greece \\ ${ }^{2}$ Department of Physiotherapy, University of Western Attica, General Hospital of Evangelismos, Greece \\ ${ }^{3}$ Department of Pediatric Orthopaedics and Neuro-orthopaedics, University of Milan, Italy \\ ${ }^{4}$ Faculty of Health Science, School of Physiotherapy, Athens Metropolitan College, Athens, Greece \\ ${ }^{5}$ Spine Department and Deformities, Interbalkan European Medical Center, Thessaloniki, Greece
}

\begin{abstract}
Horse-related athletic, recreational and professional activities is a worldwide widespread practice involving people of all ages. This kind of activity, due to the particular risks involved, is associated with a significant number of accidents and injuries of the musculoskeletal system. Spine injuries (SI) and spinal cord injuries (SCI) are serious injuries that can have devastating consequences for the rider. In the present review, we examined their epidemiology, their characteristics in terms of the mechanism and type of injury, the neurological damage they cause and the injured region of the spine. The type of the therapeutic approach and its results were also analyzed. 19 papers that included 7.569 patients, 632 (8.3\%) of whom suffered spine or spinal cord injuries, were analyzed. A total of 1.072 injuries were analyzed, $56 \%$ of which concerned men and $44 \%$ women. The fall from a horse is considered to be the main mechanism that causes injuries associated with fractures of the thoracic and lumbar spine. $58 \%$ of the patients with such injuries were hospitalized. There was a complete neurological damage in $31.8 \%$ of the patients, while in a significant proportion of $53.5 \%$ of the patients; quadriplegia was caused by the injury. Although conservative treatment in simple cases without neurological damage appears to be an effective therapeutic approach, in cases of unstable injuries and neurological impairment surgical treatment can be a method of choice with satisfactory results such as better mobilization and quality of life.
\end{abstract}

\section{Introduction}

Horse-related competition, recreational and professional activities, is a worldwide widespread practice involving people of all ages. In the US more than 30 million and in the UK about 3 million people are engaged in horse riding activities [1]. In Sweden, almost 200.000 people are related to horse riding activities and this represents the eighth most popular sport in the country [2], while in New South Wales, within a year, 43.000 people aged up to 55 years participated in organized horse riding, excluding recreational purposes [3].

However, horse riding is accompanied by a significant number of accidents with a wide range of severe injuries. Thus, these accidents may be associated with relatively harmless and easily manageable injuries or even with serious injuries with particular health consequences regarding the rider's overall quality of life. In the US, about $20 \%$ of people engaged in horse-related activities are expected to suffer some serious injury during their career. In fact, amateur equestrians have a higher incidence of injuries [4]. According to Thomas et al., a significant number of 102.904 persons were treated in US emergency departments during 2001-2003 due to non-fatal horse related injuries. This figure represents 35.7 injuries per 100,000 of population [5]. During equestrian activities, a number of deaths have been also recorded [6-9].

Due to the special circumstances in which these accidents occur and the high-energy involved in these injuries, a significant number of horse riders visit the emergency every year and part of them requires hospitalization for further treatment. Although craniocerebral injuries are the most common serious injuries which cause neurological damage and require hospitalization for further treatment, spine and spinal cord injuries represent another frequent type of injury in horse riding. It is notable that within a period of 10 years, equestrian activities were the main cause for more than $40 \%$ of sports related spinal injuries where hospitalization in the Irish National Spinal Injuries Unit was considered necessary [10]. These injuries require specialized and immediate treatment and may have significant negative effects for the health of the injured rider.

The aim of this review is to present the best available evidence on the epidemiology, the specific features, the management and the treatment of the horse-related spine and spinal cord injuries. Patients with these injuries may develop loss of function which possibly leads to a chronic disability; therefore, these injuries may have a detrimental impact on their quality of life. This review was attempted taking into account that the best of our knowledge no thorough analysis has been done till today regarding these kind of injuries as well as on the results of their treatment.

\section{Material and methods}

A thorough literature search between July 1993 and July 2018 was conducted via MEDLINE, using the following keywords: "horse riding", "horse related", "equestrian "injuries", "fractures", "accidents", "spine", "spinal fractures", and 'spinal cord". The inclusion criteria

*Correspondence to: Antonios G Angoules, Orthopaedic Department, Athens Medical Center, Athens, Greece, Tel: +30 6977011617, E-mail: antoniosangoules@yahoo.com

Key words: equestrian injuries, horse-related injuries, spine, spinal cord, fractures, epidemiology, treatment

Received: March 15, 2019; Accepted: April 08, 2019; Published: April 11, 2019 
were published papers; case series and reports, referring to (a) injuries in adults related to horses and have occurred at any time during equestrian activities and through contact with horses for various reasons and (b) spine injuries of any severity with or without a reported neurological damage. We exclude papers (a) that only recorded other injuries than those of the spine (b) that did not clearly indicate which injuries referred to the spine and (c) editorials or letters to the editor, expert opinion articles, congress proceedings and review papers.

From an initial total of 208 articles that were identified after exclusion of duplicate and non-eligible articles, title and abstract screening and addition of missed studies identified by reference list screening and citation tracking, 19 studies were found to fulfil the inclusion and exclusion criteria.

Epidemiological data on these injuries in terms of their frequency, gender, injury mechanism, type and area of injury, neurological damage caused by injuries, surgical or conservative treatment and long-term outcome of these injuries regarding the neurological state of the injured rider were analyzed in the present review.

\section{Results}

\section{Demographics}

7569 patients were included in the analysis, $632(8.3 \%)$ of which had been injured in the spine and spinal cord. The total number of spine injuries analyzed was 1.072 . These patients are included in 19 papers involving adult patients. From a total of 1.232 patients suffered an injury associated with equestrian activity, 660 (53.6\%) were men and $572(46.4 \%)$ were women. From a total of 225 patients with a spinal and spinal cord injury, $126(56 \%)$ were men and 99 (44\%) were women. From the 225 patients, 103 (54\%) had a professional engagement with horses, $71(37.2 \%)$ were riding for leisure, 11 (5.8\%) were competitive athletes and 6 (6.6\%) unknown/other (Table 1).

\section{Injury characteristics}

The mechanism related to spinal and spinal cord injuries is most frequently reported to be associated with horse-riding falls in 829 (46.9\%) cases in a total of $1769.514(29 \%)$ of these patients suffered an injury as a result of a horse kick, 91 (5.1\%) crush, 90(5\%) trample, 23 (1.3\%) bite, while in the other cases the injury of the spine was caused due to other injury mechanisms such as entrapment in the reins etc. In 94 patients with spine and spinal cord injuries, the fall was the main cause of the accident in $68(72.4 \%)$ patients, followed by hitting a stationary object in $8(8.5 \%)$ and jumping in $6(6.4 \%)$ riders. From a total of 851 spine injuries, a large proportion of $126(14.8 \%)$ were related to thoracic spine, $171(20 \%)$ thoracic spine and chest and 3 (0.4\%) the thoracolumbar junction. From the rest of the injuries, 182 $(21.4 \%)$ are recorded in the cervical spine and neck, $81(9.6 \%)$ in the lumbar spine, 58 (6.8 \%) in the back and lower spine, 45 (5.3\%) in lumbosacral, and 171 in lumbar and pelvis (20\%). In 13 (1.6\%) injuries the injured area of the spine has not been recorded, while in $1(0.1$ $\%$ ) injury a normal spinal examination is recorded. From a total of 222 recorded injuries of the spine and spinal cord, $154(69.4 \%)$ were fractures, 26 (11.7\%) were dislocations-fractures, 27 (12.1\%) were soft tissue injuries and $15(6.8 \%)$ concerned spinal cord and nerve injuries.

Regarding the neurological damage suffered by the participants in the study, from 233 horse riders for whom their neurological status was recorded after the injury, 94 (40.3\%) suffered an incomplete lesion, 56 (24\%) a complete lesion, 74 (31.8\%) haven't suffered any neurological impairment; while in $9(3.9 \%)$ the extent of the damage wasn't recorded. Regarding the type of neurological damage, 62 (53.5\%) suffered tetraplegia and 54 (46.5\%) paraplegia (Table 2).

Table 1. Demographic data I

\begin{tabular}{|c|c|c|c|c|c|c|c|}
\hline \multirow[b]{2}{*}{ Author } & \multirow[b]{2}{*}{ Patients (n) } & \multirow{2}{*}{$\begin{array}{l}\text { Patients with } \\
\text { Spinal Injuries (N) }\end{array}$} & \multirow[b]{2}{*}{ Spinal Injuries } & \multicolumn{2}{|l|}{ Age (y) } & \multicolumn{2}{|c|}{ Mean Age (y) } \\
\hline & & & & Patients (n) & $\begin{array}{l}\text { Patients with } \\
\text { Spinal Injuries (N) }\end{array}$ & Patients (n) & $\begin{array}{l}\text { Patients with } \\
\text { Spinal Injuries (N) }\end{array}$ \\
\hline Abdulkarim et al. [11] & 149 & 24 & 24 & $5-77$ & $\mathrm{n} / \mathrm{a}$ & $\mathrm{n} / \mathrm{a}$ & $\mathrm{n} / \mathrm{a}$ \\
\hline Oshima et al. [12] & 4 & $1(25 \%)$ & 1 & 30 & 30 & 30 & 30 \\
\hline Weber et al. [13] & 679 & 127 & 128 & $\mathrm{n} / \mathrm{a}$ & $\mathrm{n} / \mathrm{a}$ & $\mathrm{n} / \mathrm{a}$ & $\mathrm{n} / \mathrm{a}$ \\
\hline Oh et al. [14] & 2 & $2(100 \%)$ & 2 & $44-49$ & 47.5 & $44-49$ & 47.5 \\
\hline Altgärde et al. [2] & 141 & 15 & 15 & $19.2-70.1$ & $\mathrm{n} / \mathrm{a}$ & 33 & $\mathrm{n} / \mathrm{a}$ \\
\hline Boham et al. [15] & 1 & $1(100 \%)$ & 1 & 18 & 18 & 18 & 18 \\
\hline Papachristos et al. [16] & 172 & $78(45 \%)$ & 152 & $18-82$ & $\mathrm{n} / \mathrm{a}$ & 38 & $\mathrm{n} / \mathrm{a}$ \\
\hline Srinivasan et al. [17] & 80 & $8(10 \%)$ & 14 & $2.2-79.3$ & $\mathrm{n} / \mathrm{a}$ & 37 & $\mathrm{n} / \mathrm{a}$ \\
\hline Muller et al. [18] & 1 & $1(100 \%)$ & 1 & 37 & 37 & 37 & 37 \\
\hline Triantafyllopoulos et al. [19] & 3 & $3(100 \%)$ & 8 & $25-32$ & $25-32$ & 28 & 28 \\
\hline Lin et al. $[20]$ & 121 & $121(100 \%)$ & 121 & $0-65+$ & $0-65+$ & 37.8 & 37.8 \\
\hline Ball et al. [21] & 151 & $\mathrm{n} / \mathrm{a}$ & 35 & $20-78$ & $\mathrm{n} / \mathrm{a}$ & 47 & $\mathrm{n} / \mathrm{a}$ \\
\hline Loder RT [22] & 5033 & $123(2.4 \%)$ & $\begin{array}{l}351 \text { (Lumbar Spine } \\
\text { and Pelvis: } 171 \\
(11.9 \%) \text { Chest and } \\
\text { Thoracic spine: } 171 \\
(11.9 \%) \text { Neck and } \\
\text { cervical spine: } 9 \\
(0.6 \%)\end{array}$ & $\mathrm{n} / \mathrm{a}$ & $\mathrm{n} / \mathrm{a}$ & 39.6 & $\mathrm{n} / \mathrm{a}$ \\
\hline Yim et al. [23] & 36 & $5(13.9 \%)$ & 5 & $17-54$ & $\mathrm{n} / \mathrm{a}$ & 34 & $\mathrm{n} / \mathrm{a}$ \\
\hline Siebenga et al. [24] & 32 & $32(100 \%)$ & 36 & $8-58$ & $8-58$ & 33.7 & 33.7 \\
\hline Roe et al. [3] & 64 & $64(100 \%)$ & 64 & $0-66+$ & $0-66+$ & $\mathrm{n} / \mathrm{a}$ & $\mathrm{n} / \mathrm{a}$ \\
\hline Iba et al. [25] & 581 & $\mathrm{n} / \mathrm{a}$ & 87 & $4-81$ & $\mathrm{n} / \mathrm{a}$ & 37 & $\mathrm{n} / \mathrm{a}$ \\
\hline Chitnavis et al. [26] & 177 & $20(11.3 \%)$ & 20 & $0-80$ & $\mathrm{n} / \mathrm{a}$ & $\mathrm{n} / \mathrm{a}$ & $\mathrm{n} / \mathrm{a}$ \\
\hline Hobbs et al. [27] & 142 & $7(4.9 \%)$ & 7 & $2-74$ & $\mathrm{n} / \mathrm{a}$ & 27 & $\mathrm{n} / \mathrm{a}$ \\
\hline TOTAL & 7569 & 632 & 1072 & & & & \\
\hline
\end{tabular}

$\mathbf{n}=$ number of patients; $\mathbf{N}=$ number of patients with spinal injuries; $\mathbf{Y}=$ year; $\mathbf{n} / \mathbf{a}=$ non available 


\section{Treatment and outcome}

Out of 100 patients who reported that they needed hospitalization, $58(58 \%)$ of them were admitted to hospital for further treatment of the spine injury. The complications recorded in 4 patients were 2 cases of wound infection, complaints of osteosythesis in one case and pulmonary embolism in another case. These complications were observed in patients who underwent surgical treatment except for a patient with spinal fracture who was conservatively treated and had pulmonary embolism. Out of 76 patients where the treatment approach is referred, 22 (29\%) underwent surgical treatment for their injuries. Posterior fusion for surgical treatment of injuries was selected in 11 patients $(50 \%)$, anterior fusion in $7(32 \%)$, while for the remaining $4(18 \%)$ the surgical treatment is not mentioned. 25 patients $(33 \%)$ received conservative treatment such as early functional 19 (76\%), late functional 3 (12\%), Calcium and vitamin D supplements and Trunk Flexion Exercises 2(8\%) and 1 patient (4\%) medical corset. For the remaining 29 patients (38\%) the treatment is not mentioned. Out of 6 patients where the treatment results are reported, 3 patients (50\%) fully recovered, 2 patients $(33.4 \%)$ partially recovered and 1 patient $(16,7 \%)$ experienced neurological improvement (Tables 3 and 4 ).

\section{Discussion}

Horse riding is a worldwide widespread professional, recreational and sport activity which is associated with a significant risk of accidents and injuries of the musculoskeletal system $[3,4,10]$. Spine and spinal cord injuries are not uncommon during horse riding and equestrian activities whether for professional or other athletic or recreational purposes [1,10-20]. According to Boran et al. [10] equestrian activities over a period of 10 years in Ireland was the most common cause of spine injuries at a rate of $41,8 \%$. In fact, according to Lin et al. [20], spinal cord injury associated to horseback riding, more often results in tetraplegia followed by complete paraplegia.

The increased frequency of injuries during horse riding can be understood by taking into account that horse riding is characterized by the two-way relationship of the rider with an animal, which of course is an intelligent animal but with a different level of intelligence than

Table 2. Demographic data II

\begin{tabular}{|c|c|c|c|c|c|c|}
\hline \multirow[b]{2}{*}{ Author } & \multirow[b]{2}{*}{ Patients (n) } & \multirow{2}{*}{$\begin{array}{l}\text { Patients with Spinal } \\
\text { Injuries (n) }\end{array}$} & \multicolumn{2}{|l|}{ Gender } & \multicolumn{2}{|c|}{ Occupation - Riding Experience } \\
\hline & & & Patients (n) & $\begin{array}{l}\text { Patients with Spinal } \\
\text { Injuries (N) }\end{array}$ & Patients (n) & $\begin{array}{l}\text { (Patients with Spinal } \\
\text { Injuries (N) }\end{array}$ \\
\hline Abdulkarim et al. [11] & 149 & 24 & $\begin{array}{l}\text { M: } 62(42 \%) \mathrm{F}: 87 \\
(58 \%)\end{array}$ & & $\mathrm{n} / \mathrm{a}$ & $\mathrm{n} / \mathrm{a}$ \\
\hline Oshima et al. [12] & 4 & $1(25 \%)$ & F: $1(100 \%)$ & F: $1(100 \%)$ & $\mathrm{n} / \mathrm{a}$ & $\mathrm{n} / \mathrm{a}$ \\
\hline Weber et al. [13] & 679 & 127 & $\mathrm{n} / \mathrm{a}$ & $\mathrm{n} / \mathrm{a}$ & $\mathrm{n} / \mathrm{a}$ & $\mathrm{n} / \mathrm{a}$ \\
\hline Oh et al. [14] & 2 & $2(100 \%)$ & F:2 (100 \%) & $\mathrm{F}: 2(100 \%)$ & $\begin{array}{l}\text { Amateur Riders: } 2 \\
(100 \%)\end{array}$ & $\begin{array}{l}\text { Amateur Riders: } 2 \\
(100 \%)\end{array}$ \\
\hline Altgärde et al. [2] & 141 & 15 & M: $13(\%) \mathrm{F}: 128(\%)$ & $\mathrm{n} / \mathrm{a}$ & $\mathrm{n} / \mathrm{a}$ & $\mathrm{n} / \mathrm{a}$ \\
\hline Boham et al. [15] & 1 & $1(100 \%)$ & M:1 (100\%) & M:1 (100\%) & Rodeo Athlete:1 $(100 \%)$ & Rodeo Athlete: $1(100 \%)$ \\
\hline Papachristos et al. [16] & 172 & $78(45 \%)$ & $\begin{array}{l}\text { M:70 (41\%) F:102 } \\
(59 \%)\end{array}$ & $\mathrm{n} / \mathrm{a}$ & $\mathrm{n} / \mathrm{a}$ & $\mathrm{n} / \mathrm{a}$ \\
\hline Srinivasan et al. [17] & 80 & $8(10 \%)$ & M: $46 \%$ F: $44 \%$ & $\mathrm{n} / \mathrm{a}$ & $\mathrm{n} / \mathrm{a}$ & $\mathrm{n} / \mathrm{a}$ \\
\hline Muller et al. [18] & 1 & $1(100 \%)$ & $\mathrm{n} / \mathrm{a}$ & $\mathrm{F}: 1(100 \%)$ & $\mathrm{n} / \mathrm{a}$ & $\mathrm{n} / \mathrm{a}$ \\
\hline Triantafyllopoulos et al. [19] & 3 & $3(100 \%)$ & $\begin{array}{l}\text { M:2 (66.7\%) F:1 } \\
(33.3 \%)\end{array}$ & $\begin{array}{l}\mathrm{M}: 2(66.7 \%) \mathrm{F}: 1 \\
(33.3 \%)\end{array}$ & $\begin{array}{l}\text { Top Athletic Rider:1 } \\
(33.3 \%) \text { Professional } \\
\text { Racing Jockey:2 } \\
(66.6 \%)\end{array}$ & $\begin{array}{l}\text { Top Athletic Rider:1 } \\
(33.3 \%) \text { Professional } \\
\text { Racing Jockey:2 } \\
(66.6 \%)\end{array}$ \\
\hline Linet al. [20] & 121 & $121(100 \%)$ & $\begin{array}{l}\text { M: } 60(49.6 \%) \text { F: } 61 \\
(50.4 \%)\end{array}$ & $\begin{array}{l}\text { M: } 60(49.6 \%) \text { F: } 61 \\
(50.4 \%)\end{array}$ & $\begin{array}{l}\text { Working: } 82(67.8 \%) \\
\text { Student: } 13(10.7 \%) \\
\text { Retired: } 11(9.1 \%) \\
\text { Homemaker: } 7(5.8 \%) \\
\text { Unemployed: } 7(5.8 \%) \\
\text { Other:1 }(0.8 \%)\end{array}$ & $\begin{array}{l}\text { Working: } 82(67.8 \%) \\
\text { Student: } 13(10.7 \%) \\
\text { Retired: } 11(9.1 \%) \\
\text { Homemaker: } 7(5.8 \%) \\
\text { Unemployed: } 7(5.8 \%) \\
\text { Other:1 }(0.8 \%)\end{array}$ \\
\hline Ball et al. [21] & 151 & $\mathrm{n} / \mathrm{a}$ & $\mathrm{n} / \mathrm{a}$ & $\mathrm{n} / \mathrm{a}$ & $\begin{array}{l}\text { MeanRidingExperience } \\
=27 \text { years }\end{array}$ & $\mathrm{n} / \mathrm{a}$ \\
\hline Loder RT [22] & 5033 & $123(2.4 \%)$ & $\mathrm{n} / \mathrm{a}$ & $\mathrm{n} / \mathrm{a}$ & $\mathrm{n} / \mathrm{a}$ & $\mathrm{n} / \mathrm{a}$ \\
\hline Yim et al. [23] & 36 & $5(13.9 \%)$ & $\mathrm{M} / \mathrm{F}=8 / 1$ & $\mathrm{n} / \mathrm{a}$ & $\mathrm{n} / \mathrm{a}$ & $\mathrm{n} / \mathrm{a}$ \\
\hline Siebenga et al. [24] & 32 & $32(100 \%)$ & $\begin{array}{l}\text { M:28 (87.5 \%) F:4 } \\
(12.5 \%)\end{array}$ & $\begin{array}{l}\text { M:28 (87.5\%) F:4 } \\
(12.5 \%)\end{array}$ & $\mathrm{n} / \mathrm{a}$ & $\mathrm{n} / \mathrm{a}$ \\
\hline Roe et al. [3] & 64 & $64(100 \%)$ & $\begin{array}{l}\text { M:35 (54.7\%) F:29 } \\
(45.3 \%)\end{array}$ & $\begin{array}{l}\text { M:35 (54.7\%) F:29 } \\
(45.3 \%)\end{array}$ & $\begin{array}{l}\text { Occupational: } 21 \\
(32.8 \%) \text { Leisure: } 31 \\
(48.4 \%) \text { Competitive: } \\
7(11 \%) \text { Unknown: } 5 \\
(7.8 \%)\end{array}$ & $\begin{array}{l}\text { Occupational: } 21 \\
(32.8 \%) \text { Leisure: } 31 \\
(48.4 \%) \text { Competitive: } \\
7(11 \%) \text { Unknown: } 5 \\
(7.8 \%)\end{array}$ \\
\hline Iba et al. [25] & 581 & $\mathrm{n} / \mathrm{a}$ & $\begin{array}{l}\text { M:424 (73\%) F:157 } \\
(27 \%)\end{array}$ & $\mathrm{n} / \mathrm{a}$ & $\begin{array}{l}\text { Stable- or stud-workers: } \\
581(100 \%)\end{array}$ & $\mathrm{n} / \mathrm{a}$ \\
\hline Chitnavis et al. [26] & 177 & $20(11.3 \%)$ & $\mathrm{M} / \mathrm{F}=1 / 3$ & $\mathrm{n} / \mathrm{a}$ & $\begin{array}{l}\text { Amateurs Riders: } 44 \\
(25 \%) \text { Horse-related } \\
\text { Occupations: } 133(75 \%)\end{array}$ & $\mathrm{n} / \mathrm{a}$ \\
\hline Hobbs et al. [27] & 142 & $7(4.9 \%)$ & $\mathrm{n} / \mathrm{a}$ & $\mathrm{n} / \mathrm{a}$ & $\mathrm{n} / \mathrm{a}$ & $\mathrm{n} / \mathrm{a}$ \\
\hline TOTAL & 7569 & 632 & $\begin{array}{l}\text { M:660 (53.6\%) F:572 } \\
(46.4 \%)\end{array}$ & $\begin{array}{l}\text { M:126 (56\%) F:99 } \\
(44 \%)\end{array}$ & & \\
\hline
\end{tabular}

$\mathbf{n}=$ number of patients; $\mathbf{N}=$ number of patients with spinal injuries; $\mathbf{M}=$ male $; \mathbf{F}=$ female $; \mathbf{n} / \mathbf{a}=$ non available 
Table 3. Injury characteristics

\begin{tabular}{|c|c|c|c|c|c|c|c|}
\hline \multirow[b]{2}{*}{ Author } & \multirow[b]{2}{*}{ Mechanism of Injury } & \multirow{2}{*}{$\begin{array}{l}\text { Spinal Distribution(Spinal } \\
\text { Region) }\end{array}$} & \multicolumn{4}{|l|}{ Typy of Injury } & \multirow{2}{*}{$\begin{array}{l}\text { Neurological } \\
\text { Impairment }\end{array}$} \\
\hline & & & Fractures & $\begin{array}{l}\text { Fractures- } \\
\text { Dislocations }\end{array}$ & $\begin{array}{l}\text { Spinal Cord } \\
\& \text { Nerves }\end{array}$ & Soft Lesions & \\
\hline Abdulkarim et al. [11] & $\begin{array}{l}\text { Fall: } 23(95.8 \%) \text { Trample: } \\
1(4.2 \%)\end{array}$ & $\begin{array}{l}\text { Cervical:1 }(11.1 \%) \\
\text { Thoracic:2 }(22.2 \%) \\
\text { Thoracolumbar: } 3(66.7 \%)\end{array}$ & $\begin{array}{l}6(12.5 \%) \text { Stable:5 } \\
(83.3 \%) \text { Unstable: } 1 \\
(16.7 \%)\end{array}$ & - & - & - & $1(4.1 \%)$ \\
\hline Oshima et al. [12] & $\mathrm{n} / \mathrm{a}$ & Thoracic : $1(100 \%)$ & $\begin{array}{l}\text { T12 C ompression: } 1 \\
(100 \%)\end{array}$ & - & - & - & None: $1(100 \%)$ \\
\hline Weber et al. [13] & $\mathrm{n} / \mathrm{a}$ & $\begin{array}{l}\text { Cervical: } 38(29.7 \%) \\
\text { Thoracic: } 41(32 \%) \text { Lumbar: } \\
49 /(38.3 \%)\end{array}$ & $\mathrm{n} / \mathrm{a}$ & $\mathrm{n} / \mathrm{a}$ & $\mathrm{n} / \mathrm{a}$ & $\mathrm{n} / \mathrm{a}$ & $\mathrm{n} / \mathrm{a}$ \\
\hline Oh et al. [14] & $\begin{array}{l}\text { No Trauma History- Low } \\
\text { Bone Mineral Density: } 2 \\
(100 \%)\end{array}$ & Thoracic:2 & $\begin{array}{l}\text { T6 Compression: } 1 \text { (50 } \\
\text { \%) T7 Compression: } \\
1(50 \%)\end{array}$ & - & - & - & \\
\hline Altgärde et al. [2] & & $\mathrm{n} / \mathrm{a}$ & 3 & 1 & - & 11 & $\mathrm{n} / \mathrm{a}$ \\
\hline Boham et al. [15] & $\begin{array}{l}\text { Extreme Lumbothoracic } \\
\text { Hyperflexion: } 1(100 \%)\end{array}$ & Thoracic:1 (100\%) & $\begin{array}{l}\text { T12 Chance: } 1(100 \\
\%)\end{array}$ & - & - & - & None:1 (100 \%) \\
\hline Papachristos et al. [16] & $\mathrm{n} / \mathrm{a}$ & $\mathrm{n} / \mathrm{a}$ & $69 \%$ & $\mathrm{n} / \mathrm{a}$ & 5 & $\mathrm{n} / \mathrm{a}$ & Complete Lesion :1 \\
\hline Srinivasan et al. [17] & $\mathrm{n} / \mathrm{a}$ & $\begin{array}{l}\text { Cervical :8 }(57.2 \%) \\
\text { Thoracic: } 3(22.8 \%) \\
\text { Lumbosacral: } 3(22.8)\end{array}$ & $\begin{array}{l}8 \text { Burst: } 2 \text { Transverse } \\
\text { process: most } \\
\text { commonly }\end{array}$ & - & - & - & None:8 (100 \%) \\
\hline Muller et al. [18] & Kick: $1(100 \%)$ & Cervical: $1(100 \%)$ & - & C4/5:1 (100\%) & - & - & $\begin{array}{l}\text { ASIA A (Complete } \\
\text { Paraplegia): } 1(100 \%)\end{array}$ \\
\hline Triantafyllopoulos et al. [19] & $\mathrm{n} / \mathrm{a}$ & Thoracic: $8(100 \%)$ & $\begin{array}{l}\text { T5 : } 1(16.7 \%) \text { T6: } 2 \\
(33.3 \%) \text { T7:3 }(50 \%) \\
\text { (AO type) A:4 }(66.7 \\
\%) \text { B: } 2(33.3 \%)\end{array}$ & T6-T7: $2(100 \%)$ & - & - & $\begin{array}{l}\text { ASIA A : } 1(33.3 \%) \\
\text { ASIA B:1 }(33.3 \%) \\
\text { None: } 1(33.3 \%)\end{array}$ \\
\hline Lin et al. $[20]$ & $\mathrm{n} / \mathrm{a}$ & $\begin{array}{l}\text { Cervical: } 58(47.9 \%) \\
\text { Thoracic: } 34(28.1 \%) \\
\text { Lumbosacral: } 20(16.5 \%) \text { Not } \\
\text { recorded: } 8(6.6 \%) \text { Normal } \\
\text { exam: } 1(0.8 \%)\end{array}$ & & & & & $\begin{array}{l}\text { Paraplegia } \\
\text { incomplete: } 24 \\
(19.8 \%) \text { Paraplegia } \\
\text { complete: } 29(24.0 \\
\%) \text { Tetraplegia } \\
\text { incomplete :49 } \\
(40.5 \%) \text { Tetraplegia } \\
\text { complete: } 10(8.3 \%) \\
\text { Normal exam/minimal } \\
\text { deficit: } 5(4.1 \%) \text { Not } \\
\text { recorded:4 }(3 \%)\end{array}$ \\
\hline Ball et al. [21] & $\mathrm{n} / \mathrm{a}$ & $\mathrm{n} / \mathrm{a}$ & $25(17 \%)$ & - & $10(6 \%)$ & - & $\mathrm{n} / \mathrm{a}$ \\
\hline Loder RT [22] & $\mathrm{n} / \mathrm{a}$ & $\begin{array}{l}\text { Cervical Spine \& Neck: } 9 \\
(2.6 \%) \text { Thoracic Spine \& } \\
\text { Chest: } 171(48.7 \%) \text { Lumbar } \\
\text { \& Pelvis: } 171(48.7 \%)\end{array}$ & $\mathrm{n} / \mathrm{a}$ & $\mathrm{n} / \mathrm{a}$ & $\mathrm{n} / \mathrm{a}$ & $\mathrm{n} / \mathrm{a}$ & $\begin{array}{l}\text { Complete Lesion: } 1 \\
\text { Incomplete Lesion: } 1\end{array}$ \\
\hline Yim et al. [23] & $\mathrm{n} / \mathrm{a}$ & $\begin{array}{l}\text { Cervical: } 3(60 \%) \text { Lumbar: } \\
2(40 \%)\end{array}$ & $\begin{array}{l}\text { C5 Burst: } 1(50 \%) \\
\text { L5 Burst: } 1(50 \%)\end{array}$ & C6 : $1(100 \%)$ & - & - & $\begin{array}{l}\text { None: } 2(40 \%) \\
\text { Tetraplegia:2 }(40 \%) \\
\text { NotRecorded: } 1(20 \%)\end{array}$ \\
\hline Siebenga et al. [24] & $\mathrm{n} / \mathrm{a}$ & $\begin{array}{l}\text { Cervical:2 (5.6 \%) Thoracic: } \\
11(30.5 \%) \text { Lumbar: } 23 \\
(63.9 \%)\end{array}$ & $\begin{array}{l}\text { C5:1 (2.8 \%) C6:1 } \\
(2.8 \%) \text { T6:1 }(2.8 \\
\%) \text { T7:1 }(2.8 \%) \\
\text { T8:1 (2.8 \%) T11:1 } \\
(2.8 \%) \text { T12:7 (19.4 } \\
\%) \text { L1:14 (38.9 \% } \\
\text { L2:5 (13.9 \%) L3:3 } \\
\text { (8.4 \%) L4:1 (2.8 } \\
\%) \text { A1:15 (44.1\%) } \\
\text { A3:17 (50\%) B1:2 } \\
\text { (5.9\%) (AO type) }\end{array}$ & - & - & - & None:36 (100 \%) \\
\hline Roe et al. [3] & $\begin{array}{l}\text { Fall in Flight: } 32(54.2 \%) \\
\text { Horse and Rider Fall: } 6(10.1 \\
\%) \text { Hit Stationary Object: } 8 \\
(13.5 \%) \text { Fall from Stationary } \\
\text { Object: } 2 \text { (3.4\%) Fall from Gig: } \\
1(1.7 \%) \text { Jumping: } 6(10.2 \%) \\
\text { EquipmentFailure: } 4(6.8 \%)\end{array}$ & $\begin{array}{l}\text { Cervical: } 24 \text { (38 \%) Thoracic: } \\
17(27 \%) \text { Lumbosacral: } 22 \\
(35 \%)\end{array}$ & $\begin{array}{l}\text { Burst: } 18(45 \%) \\
\text { Wedge: } 9(22.5 \%) \\
\text { Other: } 13(32.5 \%)\end{array}$ & 21 & - & - & $\begin{array}{l}\text { Complete Lesion: } 13 \\
\text { Incomplete Lesion: } 19\end{array}$ \\
\hline Iba et al. [25] & $\mathrm{n} / \mathrm{a}$ & $\begin{array}{l}\text { Neck: } 29(33.3 \%) \text { Back and } \\
\text { lower spine: } 58(66.7 \%)\end{array}$ & $13(8.8 \%)$ & - & - & - & $\mathrm{n} / \mathrm{a}$ \\
\hline Chitnavis et al. [26] & $\mathrm{n} / \mathrm{a}$ & $\begin{array}{l}\text { Cervical:8 (40 \%) Thoracic:5 } \\
(25 \%) \text { Lumbar:7 (35\%) }\end{array}$ & 4 & - & - & 16 & None:20 (100\%) \\
\hline Hobbs et al. [27] & $\begin{array}{l}\text { Fall: } 5 \text { (71.4 \%) Kick: } 1 \\
(14.3 \%) \text { Crush: } 1(14.3 \%)\end{array}$ & $\begin{array}{l}\text { Cervical: } 1(14.3 \%) \text { Thoracic: } \\
1(14.3 \%) \text { No trecorded: } 5 \\
(71.4 \%)\end{array}$ & $\begin{array}{l}\text { C1: } 1 \text { T10: } 1 \text { Not } \\
\text { recorded: } 5\end{array}$ & $\mathrm{n} / \mathrm{a}$ & $\mathrm{n} / \mathrm{a}$ & $\mathrm{n} / \mathrm{a}$ & $\begin{array}{l}\text { Quadriplegia: } 1 \\
(14.3 \%)\end{array}$ \\
\hline
\end{tabular}

$\mathbf{n} / \mathbf{a}=$ non available 
Table 4. Treatment and outcome

\begin{tabular}{|c|c|c|c|c|c|}
\hline Author & Admissions & Length of Stay (d) & Treatment & Complications & Outcome \\
\hline Abdulkarim et al. [11] & $\mathrm{n} / \mathrm{a}$ & $\mathrm{n} / \mathrm{a}$ & $\begin{array}{l}\text { Surgical Fixation : } 1(4.2 \%) \text { Not } \\
\text { Recorded:23 (95.8 \%) }\end{array}$ & $\mathrm{n} / \mathrm{a}$ & $\mathrm{n} / \mathrm{a}$ \\
\hline Oshima et al. [12] & $1(100 \%)$ & 17 & Medical Corset:1 (100 \%) & $\mathrm{n} / \mathrm{a}$ & FullyRecovered: $1(100 \%)$ \\
\hline Weber et al. [13] & $\mathrm{n} / \mathrm{a}$ & $\mathrm{n} / \mathrm{a}$ & $\mathrm{n} / \mathrm{a}$ & $\mathrm{n} / \mathrm{a}$ & $\mathrm{n} / \mathrm{a}$ \\
\hline Oh et al. [14] & $0(0 \%)$ & & $\begin{array}{l}\text { Calcium and Vitamin D Supplements \& } \\
\text { Trunk Flexion Exercises : } 1(50 \%) \text { Calcium } \\
\text { and Vitamin D Supplements, Orthosis \& } \\
\text { Trunk Flexion Exercises : } 1(50 \%)\end{array}$ & $\mathrm{n} / \mathrm{a}$ & $\mathrm{n} / \mathrm{a}$ \\
\hline Altgärde et al. [2] & $\mathrm{n} / \mathrm{a}$ & $\mathrm{n} / \mathrm{a}$ & $\mathrm{n} / \mathrm{a}$ & $\mathrm{n} / \mathrm{a}$ & $\mathrm{n} / \mathrm{a}$ \\
\hline Boham et al. [15] & $1(100 \%)$ & & Posterior Fusion (T11-L1 + Grafts):1 (100 \%) & Wound Infection: 1 & $\begin{array}{l}\text { Return to full activity: } 1 \\
(100 \%)\end{array}$ \\
\hline Papachristos et al. [16] & $\mathrm{n} / \mathrm{a}$ & $\mathrm{n} / \mathrm{a}$ & $\begin{array}{l}\text { Surgical Fixation: } 17 \% \text { Rehabilitation } \\
\text { Therapy for Spinal Injuries: } 19 \%\end{array}$ & $\mathrm{n} / \mathrm{a}$ & $\mathrm{n} / \mathrm{a}$ \\
\hline Srinivasan et al. [17] & $\mathrm{n} / \mathrm{a}$ & 1-17 Mean: $3 \cdot 7$ & Fusion: $2(25 \%)$ & $\mathrm{n} / \mathrm{a}$ & $\mathrm{n} / \mathrm{a}$ \\
\hline Muller et al. [18] & $1(100 \%)$ & & Anterior Fusion:1 (100 \%) & $\mathrm{n} / \mathrm{a}$ & $\begin{array}{l}\text { Improvement from ASIA A } \\
\text { to ASIA D:1 }(100 \%)\end{array}$ \\
\hline Triantafyllopoulos et al. [19] & $3(100 \%)$ & $\mathrm{n} / \mathrm{a}$ & $\begin{array}{l}\text { Posterior Fusion (T3-T9, T4-T10): } 2 \text { (66.7 } \\
\% \text { ) Anterior Fusion (T5-T8) :1 (33.3\%) }\end{array}$ & $\mathrm{n} / \mathrm{a}$ & $\begin{array}{l}\text { Complete recovery: } 1(33.3 \\
\%) \text { Sensory function slightly } \\
\text { improvement: } 1(33.3 \%) \text { No } \\
\text { Neurologicalimprovement: } \\
1(33.3 \%)\end{array}$ \\
\hline Lin et al. $[20]$ & $\mathrm{n} / \mathrm{a}$ & $\mathrm{n} / \mathrm{a}$ & $\mathrm{n} / \mathrm{a}$ & $\mathrm{n} / \mathrm{a}$ & $\mathrm{n} / \mathrm{a}$ \\
\hline Ball et al. [21] & $\mathrm{n} / \mathrm{a}$ & $\mathrm{n} / \mathrm{a}$ & $\begin{array}{l}\text { Rehabilitation Therapy for Spinal Fractures: } \\
70 \% \text { Rehabilitation Therapy for Spinal Cord } \\
\text { Trauma: } 100 \%\end{array}$ & & \\
\hline Loder RT [22] & $\mathrm{n} / \mathrm{a}$ & $\mathrm{n} / \mathrm{a}$ & $\mathrm{n} / \mathrm{a}$ & $\mathrm{n} / \mathrm{a}$ & $\mathrm{n} / \mathrm{a}$ \\
\hline Yim et al. [23] & $\mathrm{n} / \mathrm{a}$ & $\mathrm{n} / \mathrm{a}$ & $\begin{array}{l}\text { Anterior Fusion:2 (66.6 \%) PosteriorFusion: } \\
1(33.3 \%)\end{array}$ & $\mathrm{n} / \mathrm{a}$ & $\mathrm{n} / \mathrm{a}$ \\
\hline Siebenga et al. [24] & $32(100 \%)$ & $\begin{array}{l}\text { Early Functional } \\
\text { Treatment: } 11.8 \\
\text { Late Functional } \\
\text { Treatment: } 43.0 \\
\text { SurgicalStabilization: } \\
17.3\end{array}$ & $\begin{array}{l}\text { Early Functional: } 19(59.4 \%) \text { Late } \\
\text { functional: } 3(9.4 \%) \text { Posterior with } \\
\text { posterolateral and transpedicular fusion:4 } \\
(12.5 \%) \text { Posterior with transpedicular } \\
\text { fusion:1 }(3.1 \%) \text { Posterior with posterolateral } \\
\text { fusion:1 }(3.1 \%) \text { Posterior without attempted } \\
\text { fusion:1 }(3.1 \%) \text { Anterior with bisegmental } \\
\text { fusion:3 }(9.4 \%)\end{array}$ & $\begin{array}{l}\text { Wound Infection: } 1 \\
\text { Complaints of } \\
\text { Osteosythesis:1 Pulmonary } \\
\text { embolism: } 1\end{array}$ & $\begin{array}{l}\text { PermanentOccupational } \\
\text { Disabilities: } 22 \%\end{array}$ \\
\hline Roe et al. [3] & $\mathrm{n} / \mathrm{a}$ & $\mathrm{n} / \mathrm{a}$ & $\mathrm{n} / \mathrm{a}$ & $\mathrm{n} / \mathrm{a}$ & $\mathrm{n} / \mathrm{a}$ \\
\hline Iba et al. [25] & $\mathrm{n} / \mathrm{a}$ & $\mathrm{n} / \mathrm{a}$ & $\mathrm{n} / \mathrm{a}$ & $\mathrm{n} / \mathrm{a}$ & $\mathrm{n} / \mathrm{a}$ \\
\hline Chitnavis et al. [26] & $42(24 \%)$ & $\mathrm{n} / \mathrm{a}$ & $\mathrm{n} / \mathrm{a}$ & $\mathrm{n} / \mathrm{a}$ & $\mathrm{n} / \mathrm{a}$ \\
\hline Hobbs et al. [27] & $\mathrm{n} / \mathrm{a}$ & $\mathrm{n} / \mathrm{a}$ & $\begin{array}{l}\text { Surgical Fixation:1 (14.3 \%) NotRecorded: } \\
6(85.7 \%)\end{array}$ & $\mathrm{n} / \mathrm{a}$ & $\mathrm{n} / \mathrm{a}$ \\
\hline
\end{tabular}

$\mathbf{n} / \mathbf{a}=$ non available

humans. The rider is 4 meters ( 13 feet) above the ground on an animal weighing up to $500 \mathrm{~kg}$, which can run at speeds up to $65 \mathrm{~km} / \mathrm{h}$ (40mph). A horse can also kick with a force of 1.8 times its bodyweight [19]. These special conditions create an increased risk of an accident. It has been estimated that horse riding or equestrian activities are more dangerous than racing and 20 times more dangerous than riding a motorcycle [1]. So, while a motorcyclist is likely to suffer a serious injury every 7,000 hours of riding, in horse riding a serious injury is expected every 350 hours of riding [21-28].

Because of these risks, spine injuries in horse riding are frequent. A study on horse riding injuries conducted in Germany showed that spine fractures seem to be as common as clavicle fractures, while another study conducted at the same country showed that thoracolumbar spine fractures represent a total of $10 \%$ and is the second frequent injury after craniocerebral injuries [24].

These findings are confirmed by this review, where a significant proportion of the patients $(8 \%)$ who suffered horse riding-related injuries had a spine injury. This review showed that spine and spinal cord injuries are more frequent in men engaged in equestrian activities. This comes into contradiction with the findings of previous studies published in the literature which refer to a higher incidence of injuries in female horse riders in relation to male riders [11,20,29]. According to Silver et al. [1], in an earlier review on spinal injuries following horse riding accidents, female riders are more likely to suffer a serious injury that requires hospitalization than men, and they believe that this is the result of the greater involvement of women in horse riding. According to Srinivasan et al. [17], the percentage of women who have suffered a neurological injury related to equestrian sports is higher and equal to $54 \%$, with the younger female riders being at greater risk.

The larger proportion regarding male patients found in this review may be due to the fact that the group of patients studied was a heterogeneous population sample. More specifically, the participants were people who have suffered a spine injury and had been engaged in horse riding for various reasons such as professional, athletic or recreational purposes. As shown in the study of Iba et al. [25], it is likely that more men are engaged professionally in equestrian activities, which also explains the greater incidence of general injuries related to equestrian activities.

Although there is a great heterogeneity that has to do with the professional involvement of people studied in this review and with the 
reason why they participated in equestrian activities, a large proportion of those who suffered injuries during horse riding were amateurs and their involvement with horse riding was just for recreational purposes, without excluding professional athletes or people who have other professional engagement with horses. However, no statistically significant differences can be found regarding the frequency of spine injuries between amateurs and professional horse riders.

The fall from a horse is considered to be the main injury mechanism during horse riding, with a rate of $18.1 \%-95.8 \%$ among the recorded injuries studied. In this study the incidence rate of musculoskeletal injuries related to horse-riding falls is found to be $46.9 \%$ of overall injuries and $72.4 \%$ of spine and spinal cord injuries, followed by hitting a stationary object and jumping. It must be noted that in many cases not only one injury mechanism is associated with the horse rider. So, the horse rider may have a fall from the horse, hit a stationary object or be trampled by his own horse or by another horse following.

Most of the injuries are recorded in the thoracic and lumbar spine. These findings are in agreement with those of an earlier review of Silver at al. [1]. The authors of this paper note that unlike other athletic activities where most of the injuries are located in the cervical spine, in horse riding most injuries are located in the lower spine and this difference is attributed to the different potential injury mechanisms. The most frequent injury mechanism for horse riders is the fall from a moving horse from a height of 3 meters above the ground. The way the horse rider falls will also determine the area of the spine that is likely to be damaged. Thus, hitting the head after falling is likely to cause a hyperflexion injury of the cervical spine in conjunction with a head injury, while sitting in the classical position with the head up in case of a fall the rider is more likely to fall onto his buttocks and thus suffer a thoracic or lumbar spine injury $[1,10,19]$. Thoraco-lumbar fractures are common injuries associated with equestrian activities. Unseated riders are likely to be forced into sudden backward movement due to tree branches or other obstacles as they ride, resulting an injury in this area [1].

In the thoracic and lumbar spine and especially in the lower thoracic spine a significant number of fractures are recorded and to a lesser extent fractures- dislocations, although the spines are covered by a large number of muscles and ligaments that offer them strong protection. The thoracic spine is protected not only by strong ligaments but also by the ribs and the sagittal orientation of the facet joints which resist the axial rotation and horizontal translation and offer protection from the injuries [30]. It is obvious that accidents such as falls from the horse are high-energy injuries that exert great pressure on the spine and can cause serious injuries. It has been postulated that thoracic spine lesions due to the special architecture of this anatomic area characterized by reduced blood supply and spinal canal narrowness are associated with paraplegia in a percentage equal to $80 \%$ [30]. This review shows that spine and spinal cord injuries when accompanied by neurological impairment mostly lead to quadriplegia and in some cases in paraplegia.

These unstable injuries often require a surgery with a potential discectomy, spinal cord decompression and spinal fusion with or without implants in order to restore the normal architecture of the spine, to preserve the neurological function and to ensure ambulation, autonomous mobility and the return of the patient to the normal activities of daily living $[15,19,30]$. Regarding the surgical treatment, the posterior surgical approach seems to be more frequent than the anterior. Stable spinal injuries may efficiently managed following early or late functional rehabilitation or brace application. Physical rehabilitation is a key component of dealing with these cases both postoperatively for the further protection of the patient but also on a longer-term basis since chronic physical difficulties often accompany these injuries.

In this study, differences in the frequency of spine injuries between professional and amateur horse riders could not be ascertained. However, it has been reported that amateur jockeys have a greater chance of falling off the horse than their professional counterparts, resulting in a higher injury frequency. Amateur jockeys are more likely to suffer a fracture and undergo surgery due to this activity than professional athletes. In a 25 -year survey on hospitalization due to horse-related injuries, most of the patients who engaged in horse riding activities at an amateur level were living in urban areas. Although it seems reasonable professional horse riders to be at greater risk due to the higher speed they develop and the larger risks they face during horse riding, however, the better training and the better quality of communication they develop with the horse, in combination with the use of more trained horses, is likely to reduce the risk of a fall and a general accident [17,31-33].

Although the usefulness of the helmet for the prevention of craniocerebral injuries is proven and suggested for all ages, especially for the younger ones $[23,28,34]$, the effectiveness of body protectors in reducing the incidence of thoracolumbar spine injuries has not yet been ascertained [20]. Although Bixby-Hammett and Brooks [35] argue that body protectors cannot protect the spine from rotation or prevent compression injuries, Hasler et al. [33], however, suggest that the use of protective waistcoats can be effective at reducing injuries during horse riding. It should be noted that wearing body protectors is not an internationally adopted practice in horse related activities, and this could have a negative impact that leads to a higher incidence of trunk and thoracic spine injuries during these activities [23] and especially to rib and soft tissue injuries. A more targeted research is needed in order to prevent these injuries and reduce their frequency and their negative consequences [7].

\section{Conclusions}

Engaging with horses for athletic, recreational, and professional purposes, due to the special circumstances of this activity, is associated with a number of accidents. These accidents can cause spine and spinal cord injuries which are the most frequent injuries. Thoracic and lumbar spine are the most common injured areas due to the frequent falls of the horse riders and the particular mechanism of injury associated with these patients. Spine fractures represent the most common injury and in a large proportion may be accompanied by a partial or complete neurological damage. Although conservative treatment in simple cases without neurological damage appears to be an effective therapeutic approach in most of the cases, in cases of unstable injuries and neurological damages surgical treatment can be a method of choice with satisfactory results. More randomized and high quality studies are required to determine the preventive measures needed in order to reduce those injuries which can have serious consequences for the quality of horse rider's life.

\section{References}

1. Silver JR, Parry JM (1991) Hazards of horse-riding as a popular sport. Br J Sports Med 25: 105-110. [Crossref]

2. Altgarde J, Redeen S, Hilding N, Drott P (2014) Horse-related trauma in children and adults during a two year period. Scand J Trauma Resusc Emerg Med 22: 40. [Crossref] 
3. Roe JP, Taylor TK, Edmunds IA, Cumming RG, Ruff SJ, et al. (2003) Spinal and spinal cord injuries in horse riding: The New South Wales experience 1976-1996. ANZ J Surg 73: 331-334. [Crossref]

4. Lang J, Sathivelu M, Tetsworth K, Pollard C, Harvey K, et al. (2014) The epidemiology of horse-related injuries for different horse exposures, activities, and age groups in Queensland, Australia. J Trauma Acute Care Surg 76: 205-212. [Crossref]

5. Thomas KE, Annest JL, Gilchrist J, Bixby-Hammett DM (2006) Non-fatal horse related injuries treated in emergency departments in the United States, 2001-2003. Br J Sports Med 40: 619-626. [Crossref]

6. Moss PS, Wan A, Whitlock MR (2002) A changing pattern of injuries to horse riders. Emerg Med J 19: 412-414. [Crossref]

7. Turner M, McCrory P, Halley W (2002) Injuries in professional horse racing in Great Britain and the Republic of Ireland during 1992-2000. Br J Sports Med 36: 403-409. [Crossref]

8. McCrory P, Turner M (2005) Equestrian injuries. Med Sport Sci 48: 8-17. [Crossref]

9. Ingemarson H, Grevsten S, Thoren L (1989) Lethal horse-riding injuries. J Trauma 29: 25-30. [Crossref]

10. Boran S, Lenehan B, Street J, McCormack D, Poynton A (2011) A 10-year review of sports-related spinal injuries. Ir J Med Sci 180: 859-863. [Crossref]

11. Abdulkarim A, Juhdi A, Coffey P, Edelson L (2018) Equestrian Injury Presentations to a Regional Trauma Centre in Ireland. Emerg Med Int 2018: 7394390. [Crossref]

12. Oshima K, Murata M1, Aoki M1, Nakajima J1, Sawada Y, et al. (2018) Report of Four Cases with Equestrian Injury: Therapeutic Approach and Outcome. Case Rep Emerg Med 2018: 8283179. [Crossref]

13. Weber CD, Nguyen AR, Lefering R, Hofman M, Hildebrand F,et al. (2017) Blunt injuries related to equestrian sports: results from an international prospective trauma database analysis. Int Orthop 41: 2105-2112. [Crossref]

14. Oh J, Oh HM, Lee JI (2016) Horseback Riding-Related Vertebral Compression Fracture from Walking in Women with Low Bone Mineral Density: Reports of Two Cases. Curr Sports Med Rep 15: 38-40. [Crossref]

15. Boham M, O'Connell K (2014) Unusual mechanism of injury resulting in a thoracic chance fracture in a rodeo athlete: a case report. J Athl Train 49: 274-279. [Crossref]

16. Papachristos A, Edwards E, Dowrick A, Gosling C (2014) A description of the severity of equestrian-related injuries (ERIs) using clinical parameters and patient-reported outcomes. Injury 45(9): 1484-1487. [Crossref]

17. Srinivasan V, Pierre C, Plog B, Srinivasan K, Petraglia AL,et al. (2014) Straight from the horse's mouth: neurological injury in equestrian sports. Neurol Res 36: 873-877. [Crossref]

18. Muller CW, Decker S, Thietje R, Krettek C (2013) Emergency closed reduction of a $\mathrm{c} 4 / 5$ fracture dislocation with complete paraplegia resulting in profound neurologic recovery. Case Rep Orthop 2013: 272865. [Crossref]

19. Triantafyllopoulos I, Panagopoulos A, Sapkas G (2013) Mid-Thoracic Spinal Injuries during Horse Racing: Report of 3 Cases and Review of Causative Factors and Prevention Measurements. Case Rep Orthop 2013: 715409. [Crossref]
20. Lin CY, Wright J, Bushnik T, Shem K (2011) Traumatic spinal cord injuries in horseback riding: a 35-year review. Am J Sports Med 39: 2441-2446. [Crossref]

21. Ball JE, Ball CG, Mulloy RH, Datta I, Kirkpatrick AW (2009) Ten years of major equestrian injury: are we addressing functional outcomes? J Trauma Manag Outcomes 3: 2. [Crossref]

22. Loder RT (2008) The demographics of equestrian-related injuries in the United States injury patterns, orthopedic specific injuries, and avenues for injury prevention. $J$ Trauma 65: 447-460. [Crossref]

23. Yim VW, Yeung JH, Mak PS, Graham CA, Lai PB, et al. (2007) Five-year analysis of Jockey Club horse-related injuries presenting to a trauma centre in Hong Kong. Injury 38: 98-103. [Crossref]

24. Siebenga J, Segers MJ, Elzinga MJ, Bakker FC, Haarman HJ, et al. (2006) Spine fractures caused by horse riding. Eur Spine $J$ 15: 465-471. [Crossref]

25. Iba K, Wada T, Kawaguchi S, Fujisaki T, Yamashita T,et al. (2001) Horse-related injuries in a thoroughbred stabling area in Japan. Arch Orthop Trauma Surg 121: 501504. [Crossref]

26. Chitnavis JP, Gibbons CL, Hirigoyen M, Lloyd Parry J et al. (1996) Accidents with horses: what has changed in 20 years? Injury 27: 103-105. [Crossref]

27. Hobbs GD, Yealy DM, Rivas J (1994) Equestrian injuries: a five-year review. J Emerg Med 12: 143-145. [Crossref]

28. Sorli JM, Equestrian injuries: a five year review of hospital admissions in British Columbia, Canada. British Medical Journal, 2000. 6(1): p. 59.

29. Sandiford N, Buckle C, Alao U, Davidson J, Ritchie J (2013) Injuries associated with recreational horse riding and changes over the last 20 years: a review. JRSM Short Rep 4: 2042533313476688 . [Crossref]

30. Shapiro S, Abel T, Rodgers RB (2002) Traumatic thoracic spinal fracture dislocation with minimal or no cord injury. Report of four cases and review of the literature. $J$ Neurosurg 96: 333-337. [Crossref]

31. Rueda MA, Halley WL, Gilchrist MD (2010) Fall and injury incidence rates of jockeys while racing in Ireland, France and Britain. Injury 41: 533-539. [Crossref]

32. Abu-Kishk I, Klin B, Gilady-Doron N, Jeroukhimov I, Eshel G (2013) Hospitalization due to horse-related injuries: has anything changed? A 25 year survey. Isr Med Assoc $J$ 15: 169-172. [Crossref]

33. Hasler RM, Gyssler L, Benneker L, Martinolli L, Schötzau A, et al. (2011) Protective and risk factors in amateur equestrians and description of injury patterns: A retrospective data analysis and a case - control survey. J Trauma Manag Outcomes 5: 4. [Crossref]

34. Angoules G, Angoules N, Angoules A (2017) A Review of Incidence and Injury Patterns of Equestrian-Related Accidents in Children and Adolescents. Br J Med Med Res 21: 1-7.

35. Bixby-Hammett D, Brooks WH (1990) Common injuries in horseback riding. A review Sports Med 9: 36-47. [Crossref]

Copyright: (C2019 Angoules AG. This is an open-access article distributed under the terms of the Creative Commons Attribution License, which permits unrestricted use, distribution, and reproduction in any medium, provided the original author and source are credited. 